INSULIN RESISTANCE AND POLYCYSTIC OVARY SYNDROME

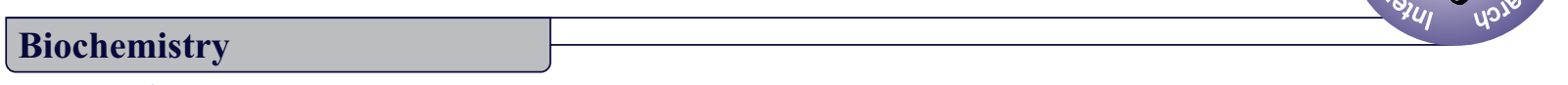

Dr. Vanita Senior Demonstrator, Biochemistry department, Dr.S.N. medical college and associated Sonagara*

Dr. Priyanka Mor

\section{Anjana Kanwar} Rathore group of hospital, Jodhpur. *Corresponding Author

Senior Demonstrator, Biochemistry department, Dr.S.N. medical college and associated group of hospital, Jodhpur.

Senior Demonstrator, Biochemistry department, Dr.S.N. medical college and associated group of hospital , Jodhpur

\title{
ABSTRACT
}

INTRODUCTION: To find out the level of insulin and insulin resistance in polycystic ovary syndrome. MATERIAL AND METHODS: The present study was conducted on 30 Polycystic Ovarian Syndrome female patients of age group (15-20, 20-30 and 30-40years) attending the Out Patient Clinics of Department of Obstetrics and Gynaecology, Dr. S.N. Medical College and its associated group of Hospitals, Jodhpur. Glucose, insulin and insulin resistance biochemical parameters were analyzed by commercially available reagents and kits using semi auto and fully auto analyzers. RESULTS: we have found a very significant and statically significant change in insulin and insulin resistance in polycystic ovary syndrome in comparison to controls .CONCLUSION: Regular evaluation of Blood Sugar, Insulin, Insulin Resistance routinely in PCOS patients that may have utility of complication, risk prediction and diagnostic role in the early detection of metabolic abnormalities, endocrine derangements and active and timely management of these alterations can prevent the risk squeal of co-morbid conditions of development of Diabetes and Cardiovascular diseases in Polycystic Ovarian Syndrome females.

\section{KEYWORDS}

\section{PCOS, Cardiovascular Disease, Diabetes, Insulin.}

INTRODUCTION: The prevalence of polycystic ovarian syndrome among adolescents and young girls in India was $22.5 \%$ by Rotterdam and $10.7 \%$ by Androgen Excess Society criteria ${ }^{1}$ The short term consequences of PCOS include irregular menses, Obesity, infertility, Hirsutism/acne/androgenic alopecia, glucose intolerance/acanthosis nigricans and long term deleterious effects of PCOS are Diabetes Mellitus $^{2}$ PCOS does not cause anovulation rather; PCOS is the consequence of chronic anovulation ${ }^{3}$ The principle features of PCOS are insulin resistance and hyperandrogenism and the role of obesity as a contributing factor in the development of PCOS is widely acceptedand particularly the abdominal phenotype (central obesity) may be responsible for IR and associated hyperinsulinemia in women with PCOS. ${ }^{4,5}$ In View of this present study was designed to assess various contributing biochemical risk factors and the early diagnostic role of these biochemical parameters in the PCOS subject with an aim to explore the utility of these investigations in the early diagnosis and better management of PCOS and its related complications.

MATERIALAND METHODS: The present study was conducted on 30 Polycystic Ovarian Syndrome female patients of age group (15-20, 20-30 and 30-40years) attending the Out Patient Clinics of Department of Obstetrics and Gynaecology, Dr. S.N. Medical College and its associated group of Hospitals, Jodhpur. All the investigation work was performed in the Department of Biochemistry, Dr. S.N. Medical College Jodhpur. The results were compared with age matched 25 healthy control female subjects. After an overnight fast of 10-12 hours, venous blood sample was drawn from anticubital vein of each subject by using standard aseptic techniques. Following biochemical parameters were analyzed by commercially available reagents and kits using semi auto and fully auto analyzers. Glucose, insulin and insulin resistance biochemical parameters were analyzed by commercially available reagents and kits using semi auto and fully auto analyzers.

RESULTS: we have found a very significant and statically significant change in insulin and insulin resistance.

\begin{tabular}{|l|l|l|l|}
\hline Groups & $\begin{array}{l}\text { Fasting blood } \\
\text { glucose(mg/dI) }\end{array}$ & $\begin{array}{l}\text { Serum Insulin } \\
(\boldsymbol{\mu} \mathbf{I U} / \mathbf{m L})\end{array}$ & $\begin{array}{l}\text { Insulin } \\
\text { Resistance }\end{array}$ \\
\hline Healthy control $\mathrm{n}=$ & $82.97 \pm 6.61$ & $15.499 \pm 4.732$ & $3.205 \pm 1.111$ \\
25 & {$[72.26-99.34]$} & {$[9-23.79]$} & {$[1.399-5.529]$} \\
\hline PCOS Patients (30) & $\begin{array}{l}94.41 \pm 10.65 \\
{[77-110.34]}\end{array}$ & $\begin{array}{l}34.528 \pm 8.051 \\
{[19.67-48.34]}\end{array}$ & $\begin{array}{l}8.102 \pm 2.207 \\
{[4.62-13.72]}\end{array}$ \\
\hline p-value & 0.0001 & 0.0001 & 0.0001 \\
\hline
\end{tabular}

DISCUSSION: Polycystic Ovarian Syndrome (PCOS) is an endocrine reproductive metabolic syndrome affecting women of all reproductive age. It has multiple components with significant and diverse clinical implications including psychological (impaired quality of life, increased anxiety and depression), reproductive (hyperandrogenism, hirsutism, menstrual irregularity, anovulation, infertility and increased Pregnancy complications) and metabolic (impaired glucose tolerance, hyperinsulinemia, insulin resistance, type 2 diabetes mellitus and cardiovascular disease) squeal ${ }^{6,7}$.

Insulin resistance is proposed as a key pathophysiological feature of PCOS contributing to both metabolic disturbances and reproductive abnormalities. Evidence suggests that women with PCOS have a greater predisposition of obesity which may aggravate PCOS related intrinsic insulin resistance and associated reproductive and metabolic disturbances. ${ }^{8}$ PCOS is also identified as a significant non- modifiable risk factor associated with type2 diabetes mellitus by the International Diabetes Federation and women with PCOS are also proposed to have a more rapid conversion from impaired glucose tolerance to type2 diabetes mellitus. ${ }^{9}$ Further women with PCOS have an elevated prevalence to the metabolic syndrome and are at increased risk for type 2 diabetes mellitus and cardiovascular disease. In view of this, present study was carried out to evaluate plasma glucose, insulin, insulin resistance in Polycystic Ovarian Syndrome patients to find out the prevalence of various risk factors in Polycystic Ovarian Syndrome. Our results are in agreement with other study in which a significant increase in fasting sugar $(\mathrm{p}<0.0001)$, fasting insulin $(\mathrm{p}<0.0001)$, HOMA-IR $(p<0.0001)$ was observed in women with Polycystic Ovarian Syndrome as compared to healthy women. ${ }^{10}$

Researcher had also reported that plasma glucose $(\mathrm{p}<0.0001)$ and serum insulin concentrations increased significantly in PCOS subjects. ${ }^{11}$ Women with PCOS are at significantly increased risk for impaired glucose tolerance and type 2 diabetes $(31.1 \%$ impaired glucose tolerance, $7.5 \%$ undiagnosed diabetes) A statistically highly significant increase in serum insulin was observed in Polycystic Ovarian Syndrome subjects $(t=10.409, \mathrm{p}<0.0001)$ when results were compared with healthy control subjects.

Our results are in accordance with another study they also observed a highly significant increase in fasting serum insulin and insulin resistance in Polycystic Ovarian Syndrome subjects as compared with healthy control subjects. A high incidence of hyperprolactinemia was found in infertile women with PCOs . A highly significant increase in the mean insulin resistance was observed in Polycystic Ovarian Syndrome subjects $(\mathrm{t}=10.071, \mathrm{p}<0.0001)$ in comparison to healthy control subject. ${ }^{12}$

Our results are in agreement with the study of others in which a significant increase in fasting insulin $(\mathrm{p}<0.001)$, HOMA-IR $(\mathrm{p}<0.001)$ 
was observed in female suffering from Polycystic Ovarian Syndrome as compared to healthy women. Impaired glucose tolerance, Hyperinsulinemia, insulin resistance and hormonal imbalance are key pathophysiological features of PCOS contributing to both metabolic and reproductive disturbances. ${ }^{13}$

CONCLUSION: Therefore, the present study provides us an evidence and outcome that there is a need of regular evaluation of Body Mass Index, Blood Sugar, Insulin, Insulin Resistance routinely in PCOS patients that may have utility of complication, risk prediction and diagnostic role in the early detection of metabolic abnormalities, endocrine derangements and active and timely management of these alterations can prevent the risk squeal of co-morbid conditions of development of Diabetes and Cardiovascular diseases in Polycystic Ovarian Syndrome females. Appropriate prospective studies with large sample size are necessary to directly assess this.

\section{REFERENCES:}

1. Kovacs, Gabor T, Norman, Robert. Polycystic Ovary Syndrome. Cambridge University Press 2007; 37:

2. Norman RJ, Masters L, Milner CR, Wang JX, Davies MJ. Relative risk of conversion from normo-glycaemia to impaired glucose tolerance or non-insulin dependent diabetes mellitus in polycystic ovarian syndrome. Hum Reprod 2001; 16: 1995.

3. Olefsky JM, Revers RR, Prience M, et al. Insulin resistance in non insulin dependent and insulin dependent diabetes mellitus. Ads Ezp Med Biol 1958; 189: 176-205.

4. Pietro Scicchitano, Ilaria Dentamaro, Rosa Carbonara, Gabriella Bulzis, Annamaria Dachille, Paola Caputo, Roberta Riccardi, Manuela Locorotondo, Cosimo Mandurino, Marco Matteo Ciccone. Cardiovascular Risk in Women With PCOS. Int J Endocrinol Marco Matteo Ciccone. Cardiov
Metab 2012;10(4):611-618.

5. Rizzo M, Berneis K, Spinas G, Rini GB, Carmina E. Long-term consequences of polycystic ovary syndrome on cardiovascular risk. Fertil Steril. 2009 Apr;91(4 Suppl):1563-1567.

6. Schroder AK, Tauchert S, Ortmann O, Diedrich K, Weiss JM. Insulin resistance in patients with polycystic ovary syndrome. Ann Med 2004; 36: 426-439.

7. Shaw LJ, Bairey Merz CN, Azziz R, Stanczyk FZ, Sopko G, Braunstein GD, et al. Postmenopausal women with a history of irregular menses and elevated androgen measurements at high isk for worseng measurents from the National Institutes of Health--National Heart, Lung, and Blood Institute sponsored Women's Ischemia Syndrome Evaluation. J Clin Endocrinol Metab. 2008 Apr;93(4):1276-1284.

8. Azziz R, Carmina E, Dewailly D, Diamanti-Kandarakis E, Escobar-Morreale HF, Futterweit W, et al. Positions statement: criteria for defining polycystic ovary syndrome as a predominantly hyperandrogenic syndrome: an Androgen Excess Society guideline. J Clin Endocrinol Metab. 2006 Nov; 91(11): 4237-4245.

9. Moran L, Teede H. Metabolic features of the reproductive phenotypes of polycystic ovary syndrome. Hum Reprod 2009; 15:477-488.

10. Thathapudi S, Kodati V, Erukkambattu J, Katragadda A, Addepally U, Hasan Q. Anthropometric and Biochemical Characteristics of Polycystic Ovarian Syndrome in Anthropometric and Biochemical Characteristics of Polycystic Ovarian Syndrome in South Indian Women Using AES-2006 Criteria. Int J Endocrinol Metab. 2014 Jan
5;12(1):e12470. doi: 10.5812/ijem.12470. PMID:24696694; PMCID: PMC3968989.

11. Dehdashtihaghighat S, Abolfazl Mehdizadehkashi, Amirmohsen Arbabi, Mohadeseh Pishgahroudsari, Shahla Chaichian. Assessment of C-reactive Protein and C3 as Inflammatory Markers of Insulin Resistance in Women with Polycystic Ovary Syndrome: ACase-Control Study. J Reprod Infertil 2013; 14(4): 197-201.

12. Sharma N, Baliarsingh S, Kaushik GG. Biochemical association of hyperprolactinemia with hypothyroidism in infertile women. Clin Lab. 2012:58(7-8):805-10. PMID: 22997982.

13. Sharma P, Gupta A, Purohit P. Cardiovascular Risk Factors Insulin Resistance [IR], High Sensitivity C-reactive Protein (hs-CRP) and Fibrinogen in Pre- Menopausal women with Poly cystic ovarian syndrome (PCOS). Journal of Cardiovascular Disease
witival with Poly cystic ovarian
Research $2015 ; 6: 67-71$ 